\title{
COMBINATORIAL PROBLEMS ON SERIES-PARALLEL GRAPHS
}

\section{K. Takamizawa}

Central Research Labs., Nippon Electric Co. Ltd. Kawasaki, Japan 213

T. Nishizeki and N. Saito

Department of Electrical Communications

Faculty of Engineering, Tohoku University

Sendai, Japan 980

Abstract. We show, in a unified manner, that there exist linear time algorithms for many combinatorial problems defined on the class of series-parallel graphs. These include (i) the decision problem, and (ii) the minimum edge (vertex) deletion problem both with respect to a property characterized by a finite number of forbidden graphs, and (iii) the generalized matching problem.

\section{Introduction}

A large number of combinatorial problems defined on graphs are NP-complete, and hence there is probably no polynomial-time algorithr for any of them [1]. A number of such problems can be formulated as a "minimum edge (vertex) deletion problem" with respect to some graph property $\mathbb{Q}$. The problem asks a minimum number of edges (vertices) of a given graph whose deletion results in a graph satisfying Q. Various other problems can be formulated as a "generalized matching problem", in which one would like to find a maximum number of vertex-disjoint copies of a fixed graph $B$ contained in an input graph. Some systematic approaches have been achieved to these problems. Krishnamoorthy et al. have shown that the minimum vertex deletion problem is NP-complete whenever property $Q$ is nontrivial and hereditary $[12,15,23]$. They have also shown that several minimum edge deletion problems are NP-complete. It should be noted that a hereditary property $Q$ can be characterized by (possibly an infinite number of) "forbidden (induced) subgraphs", that is, a graph G 
satisfies $\mathbb{Q}$ if and only if $G$ contains none of the forbidden graphs as an (induced) subgraph $[3,11,15]$. On the other hand Kirkpatrick and Hell [13] have shown that any generalized matching problem is NP-complete if the graph $B$ has a component with at least three vertices.

Some of the combinatorial problems which are NP-complete for general graphs remains so even for a restricted class of graphs [7]. However it has been shown by ad hoc methods that polynomial-time algorithms are available for some combinatorial problems on special classes of graphs, such as planar graphs, regular graphs, bipartite graphs, or series-parallel graphs. An example is the maximum cut problem, which is NP-complete for nonplanar graphs, but there exists a polynomial-time algorithm for planar graphs, as shown by Hadlock [9].

In this paper we consider a special class of graphs, called "series-parallel graphs", which can be constructed by recursively applying "series" and "parallel" connections. The class of such graphs, which is a well known model of series-parallel electrical networks, is a restricted class of planar graphs. It has been known that many practical problems defined on such graphs can be efficiently solved, for example, "resistance of electrical networks", "reliability of systems", and "scheduling" $[4,16,17]$. The following question naturally arises: do there exist polynomial-time algorithms for all combinatorial problems defined on such a class of graphs? One can easily observe that not every combinatorial problem is polynomial-time computable even if restricted to series-parallel graphs. However we show, in a unified manner, that a number of combinatorial problems are linear time computable for series-paraliel graphs. Such a rather broad class of problems includes:

(i) the decision (i.e. yes-no) problem with respect to any property $Q$ characterized by a finite number of "forbidden (induced or homeomorphic) subgraph", in which one would like to decide whether an input graph satisfies $Q$;

(ii) the minimum edge (vertex) deletion problem with respect to the same property as above; and

(iii) the generalized matching problem. (The proof for this problem is omitted.)

Hence the following problems among others prove to be linear time computable for the class of series-parallel graphs :

(1) the minimum vertex cover problem (equivalently the maximum independent vertex set problem); 
(2) the maximum (induced) Iine-subgraph problem;

(3) the minimum edge (vertex) deletion problem with respect to property "without cycles (or paths) of specified length $n$ or any lengh $\leq \mathrm{n}$;

(4) the maximum outerplanar (induced) subgraph problem;

(5) the minimum feedback vertex set problem;

(6) the maximum ladder (induced) subgraph problem $\left(\mathrm{K}_{2,3}\right.$ and its dual are the forbidden homeomorphic subgraphs of a ladder graph [20]);

(7) the minimum path cover problem (in which one would like to find a minimum number of disjoint paths which contain all the vertices of a given graph);

(8) the maximum matching problem; and

(9) the maximum disjoint triangle problem.

Some of these problems have individually been shown to be polynomial-time computable for the class of series-parallel graphs or some larger class containing all such graphs $[2,5,6,9,22]$.

\section{Preliminary}

A multigraph $G=(V, E)$ consists of a finite set $V$ of vertices and a finite multiset $E$ of edges, each of which is a pair of distinct vertices [10]. Throughout this paper we simply call them graphs since we consider only multigraphs. A graph $G^{\prime}=\left(V^{\prime}, E^{\prime}\right)$ is a subgraph of another $G=(V, E)$ if $V^{\prime}$ is a subset of $V$ and $E^{\prime}$ is a subset of $E$. If $V^{\prime}=V, G^{\prime}$ is denoted by $G^{\prime}=G-E_{S}$ where $E_{S}=E-E^{\prime}$. We write $G^{\prime} \subset G$ if $G^{\prime}$ is a subgraph of $G$. For any subset $W$ of the vertices of a $g r a p h G$, the induced subgraph on $W$ is the maximal subgraph of $G$ with vertex set $W$, and is denoted by $G-V_{S}$ where $V_{S}=V-W$.

Two edges of a graph are series if they are incident to a vertex of degree 2, and are parallel if they join the same pair of aistict vertices. A series-parallel graph is defined recursively as follows [4]:

Definition 1. A graph consisting of two vertices joined by two parallel edges is series-parallel. If $G$ is a series-parallel graph, then a graph obtained from $G$ by replacing any edge of $G$ by series or parallel edges is series-parallel.

Definition 2. A graph $G=(V, E)$ is called a 2-terminal graph when 
two distinct vertices of $V$ are distinguished from the other vertices and also from each other, and are called the first terminal and the second terminal, respectively. We write $G=(V, E, x, y)$ if $x$ is the first terminal of $G$ and $y$ the second.

A 2-terminal graph may have one or two virtual terminals, called dummy $i(i=1,2)$, which are necessarily isolated, and distinguished from a usual terminal. They will play a special role in the "connections" or "separations" of the succeeding sections. When we wish to discriminate a non-virtual vertex from virtual one, we call it a real vertex. An underlying graph of a 2-terminal graph $G$ is a graph consisting of all edges and all real vertices of $G$.

we will introduce a "2-terminal series-parallel graph", which is slightly different from a series-parallel graph. We define two kinds of connections of 2-terminal graphs, which are used to construct 2-terminal series-parallel graphs, as follows.

Definition 3. Let two 2-terminal graphs $\mathrm{H}=\left(\mathrm{V}_{\mathrm{H}}, \mathrm{E}_{\mathrm{H}}, \mathrm{a}, \mathrm{b}\right)$ and $\mathrm{K}=\left(\mathrm{V}_{\mathrm{K}}, \mathrm{E}_{\mathrm{K}}, \mathrm{c}, \mathrm{d}\right)$ have no vertex in common: $\mathrm{V}_{\mathrm{H}} \cap \mathrm{V}_{\mathrm{K}}=\varnothing$.

(a) $H$ and $K$ are series-connectable (in type I) if both the second terminal $b$ of $H$ and the first terminal $c$ of $k$ are real. By a series connection (of type $I$ ) of 2-terminal graphs $H$ and $K$, we mean the 2-terminal graph $G_{S}$ obtained from $H$ and $K$ by identifying $b$ of $H$ with $c$ of $k$. Note that the resultant graph $G_{S}$ is regarded as a 2-terminal graph with terminals a and $d$.

(b) $H$ and $K$ are parallel connectable if both $a$ and $c$ are either real or virtual, and both $b$ and $d$ are also either real or virtual. By a parallel connection of $H$ and $K$, we mean the 2-terminal graph $G_{p}$ obtained from $H$ and $k$ by identifying $a$ with $c$ and $b$ with $d$; the terminal vertices of $H$ are also the terminal vertices of the composite graph.

If a 2-terminal graph $G_{s}$ (or $G_{p}$ ) is obtained from two 2-terminal graphs $H$ and $K$ by $a$ series connection of type $I$ (or parallel connection), we write $G_{S}=H^{*} K$ (or $G_{p}=H / / K$ ), and say that $H$ and $K$ are series separations of type $I$ of $G_{s}$ (or parallel separations of $\mathrm{G}_{\mathrm{p}}$ ).

A two-terminal series-parallel (TTSP) graph is recursively defined as follows :

Definition 4. [Two-Terminal Series-Parallel Graphs] 
(i) A two-terminal graph consisting of two vertices joined by a single edge is TTSP, and is called a minimum series-parallel graph denoted by $G_{\text {min }}$.

(ii) If $H$ and $K$ are TTSP graphs, the two terminal graphs $H^{*} K$ and $\mathrm{H} / / \mathrm{K}$ are TTSP.

It is known that every series-parallel graph is TTSP if one designates appropriate vertices, for example, two ends of an edge, as the terminals [4].

\section{Decision Problems on TTSP Graphs}

In this section we consider a decision (i.e. yes-no) problem with respect to property 0 for TTSP graphs, in which one would like to determine whether a given TTSP graph satisfies or not. We will show that whenever $Q$ can be characterized by a finite number of forbidden (induced) subgraphs, the decision problem is linear time computable.

Throughout this paper we often denote by $Q$ itself the set of all (2-terminal) graphs satisfying property $Q$, so that $G=Q$ if and only if $G$ satisfies property $\mathbb{Q}$. We employ "divide-and-conquer" [1] based on the recursive definition of a TTSP graph to solve our problems on TTSP graphs. The requirements for the success and efficiency of the method is formulated as follows.

LEMMA 1. Let $Q$ be a property defined on 2-terminal graphs. The decision problem with respect to $Q$ is linear time computable for TTSP graphs if there exists a finite set of properties $\mathbb{R}=\left\{Q_{1}, Q_{2}, \ldots \mathbb{Q}_{k}\right\}$ such that

(i) $Q \subseteq \mathbb{P}$;

(ii) it is decidable whether $G_{m i n}=\mathbb{Q}_{r}$ or not for each property $\mathbb{Q}_{\mathbf{r}} \in \mathbb{R} ;$ and

(iii) for each property $\mathbb{Q}_{\mathrm{r}} \leqslant \mathbb{P}$, there exist two sets of properties $\quad\left\{\mathbb{Q}_{\mathrm{h} 1}, \mathbb{Q}_{\mathrm{h} 2}, \ldots, \mathbb{Q}_{\mathrm{ht}}\right\} \in \mathbb{P} \quad$ and $\quad\left\{\mathbb{Q}_{\mathrm{k} 1}, \mathbb{Q}_{\mathrm{k} 2}, \ldots\right.$, $\left.\mathbb{Q}_{\mathrm{kt}}\right\} \mathbb{\mathbb { R }}$ such that

$$
\mathrm{G}_{\mathrm{T}} \in \mathbb{Q}_{\mathrm{r}} \text { iff } \underset{i=1}{\mathrm{v}}\left(\left(\mathrm{H} \in \mathbb{Q}_{\mathrm{h} i}\right) \wedge\left(\mathrm{K} \in \mathbb{Q}_{\mathrm{k} i}\right)\right),
$$

for any 2-terminal graph $\mathrm{G}_{\mathrm{T}}$ satisfying $\mathrm{G}_{\mathrm{T}}=\mathrm{H}^{*} \mathrm{~K}$ for $\mathrm{H} / / \mathrm{K})$, where * denotes a series connection of type $I, \Lambda$ denotes "and", and $v$ "or". 
Proof. Let $G=(V, E, x, y)$ be a given TTSP graph. Consider an extended decision problem in which one would like to determine for every $Q_{r}=\mathbb{P}$ whether $G=Q_{r}$ or not. Since $Q \in \mathbb{P}$ by (i), the new problem includes the original one. Therefore it is sufficient to verify that the extended decision problem is linear time computable. We will show that the recursive algorithm shown below solves this problem in linear time of the number of edges of $G$.

procedure $\operatorname{TEST}(G)$ :

if $\mathrm{G}=\mathrm{G}_{\mathrm{min}}$ then determine $\mathrm{G}_{\min }=Q_{\mathrm{r}}$ for each $Q_{\mathrm{r}} \in \mathbb{P}$

else if $G=H^{*} K$ for $H$ and $K$ both having fewer edges than $G$

then TEST $(H)$ and TEST $(R)$, and determine $G \in \mathbb{Q}_{r}$ for each $Q_{r} \in \mathbb{P}$ by using the solutions to $H$ and $K$ and condition (iii) of Lemma 1

else let $G=H / / K$ for $H$ and $K$ both having fewer edges than $G$; TEST $(H)$, TEST $(K)$, and determine $G=\mathbb{Q}_{Y}$ for each $\mathbb{Q}_{r}=\mathbf{R}$ by using the solutions to $H$ and $K$ and condition (iii) of Lemma 1

First it should be noted that any TTSP graph $G$ with e=|E| edges can be constructed from e copies of the minimum series-parallel graph $G_{\text {min }}$ by a sequence of series and parallel connections, and that such a sequence can be determined by constructing a binary decomposion tree of $G$ in $O(e)$ time $[8,19,21]$. That is, the total amount of time required for series and parallel separations is $O(e)$. Hence we shall verify that the other operations require at most o(e) time, Let $\mathrm{T}(\mathrm{e})$ denote the total amount of time required for these operations to solve the extended decision problem on $G$ with e edges. By induction on $e$ we prove that $T(e) \leq c_{1} e-c_{2}$ for some constants $c_{1}$ and $c_{2}$. If $e=1$, that is, $G=G_{m i n}$, then condition (ii) implies that one can determine in a constant time whether $G=Q_{r}$ for $\mathrm{r}=1,2, \ldots, \mathrm{k}$. Thus $\mathrm{T}(1) \leq \mathrm{c}_{1} \cdot 1-\mathrm{c}_{2}$ for appropriate constants $\mathrm{c}_{1}$ and $c_{2}$. If $e \geq 2$, then $G=H^{*} K$ or $H / / K$ for two TTSP graphs $H$ and $K$ both with fewer edges than G. Condition (iii) implies that the solution to $\mathrm{G}$ can be obtained by combining the solutions to $\mathrm{H}$ and $\mathrm{K}$ in a constant time. Note that both $k$ and $t$ are constants independent of the size $e$ of the problem instance. Thus, if $H$ has $e_{H}$ edges and $K e_{K}$ edges, then

$T(e) \leq T\left(e_{H}\right)+T\left(e_{K}\right)+k t$,

where $e_{H}, e_{K} \geq I$ and $e=e_{H}+e_{K}$. The inductive hypothesis implies

$T\left(e_{H}\right) \leq c_{1} e_{H}-c_{2}$ and $T\left(e_{K}\right) \leq c_{1} e_{K}-c_{2}$. 
Therefore, by appropriately selecting $c_{1}$ and $c_{2}$, we have $T(e) \leq$ $c_{1} e-c_{2}$, which completes the proof.

We need some more definitions.

Definition 5. Let $G=(V, E)$ be a graph, and let $x$ and $y$ be distinct vertices of $V$. A 2-terminal graph $G_{T}$ is a terminal-attached graph of $G$ if $G_{T}$ is one of the following:

(i) $\mathrm{G}_{\mathrm{T}}=(\mathrm{V}, \mathrm{E}, \mathrm{x}, \mathrm{y})$;

(ii) $\mathrm{G}_{\mathrm{TP}}=$ (Vu\{ dummy 1$\}, \mathrm{E}$, dummy $\left.1, \mathrm{y}\right)$;

(iii) $\mathrm{G}_{\mathrm{T}}=$ (Vut dummy 2 \}, $\mathrm{E}, \mathrm{x}$, dummy 2 ); and

(iv) $G_{\mathrm{rP}}=$ (Vu\{dummy 1, dummy 2), E, dummy 1, dummy 2), where dummy 1 and dummy 2 are virtual vertices.

Let $G_{T}=(V, E, x, y)$ and $G_{T}{ }^{\prime}=\left(V^{\prime}, E^{\prime}, x^{\prime}, Y^{\prime}\right)$ be any two 2-terminal graphs. $G_{T}$ ' is a 2-terminal subgraph of $G_{T}$ if (i) the underlying graph of $G_{T}$ ' is a subgraph of the underlying graph of $G_{T}$, (ii) $x^{\prime}=x$ if $x \in V^{\prime}$, otherwise $x^{\prime}$ is an isolated virtual terminal dummy 1 , and (iii) $y^{\prime}=y$ if $y^{\prime} V^{\prime}$, otherwise $y^{\prime}$ is an isolated virtual terminal dummy 2. If $V^{\prime}=V$, then we write $G_{T}{ }^{\prime}=G_{T}-E_{S}$ with $E_{s}=E^{\prime}-E^{\prime}$. If $E^{\prime}$ contains all the edges of $E$ with both ends in $V^{\prime}, G_{T}^{\prime}$ is a 2-terminal induced subgraph of $G_{T}$. If $V_{s} c V$, then $G_{T}-V_{S}$ denotes the induced subgraph $G_{T T}$ ' with vertex set $V^{\prime}$ consisting of all vertices in $V-v_{s}$ together with dummy $i(i=1$ or 2$)$ if $x($ or $y) \leqq V_{S^{*}}$ We write again $G_{T}{ }^{\prime} \subset G_{T}$ if $G_{T}{ }^{\prime}$ is a 2-terminal subgraph of $\mathrm{G}_{\mathrm{T}}$.

Definition 6. Let $\mathrm{H}=\left(\mathrm{V}_{\mathrm{H}}, \mathrm{E}_{\mathrm{H}}, \mathrm{a}, \mathrm{b}\right)$ and $\mathrm{K}=\left(\mathrm{V}_{\mathrm{K}}, \mathrm{E}_{\mathrm{K}}, \mathrm{c}, \mathrm{d}\right)$ be two 2-terminal graphs having no vertex in common: $\mathrm{V}_{\mathrm{H}} \cap \mathrm{V}_{\mathrm{K}}=\phi$. $\mathrm{H}$ and $\mathrm{K}$ are series-connectable in type II if both $b$ and $c$ are virtual. By a series connection of type II we mean the 2-terminal graph $G_{\mathbf{s}}$ obtained from the union of $H$ and $K$ by deleting $b$ and $c$. That is,

$$
G_{S}=\left(V_{H} \cup V_{K}-\{b, c\}, E_{H}+E_{K}, a, d\right) \text {. }
$$

$H$ and $K$ are called series separations of type II of $G_{S}$.

If $G_{S}$ is a series connection of $H$ and $K$, then we write, from now on, $G_{S}=H * K$ regardless of type $I$ or $I I$.

Suppose that property $Q$ is defined on graphs by a finite set of forbidden subgraphs $B=\left\{B_{1}, B_{2}, \ldots, B_{q}\right\}$. Thus $G \in Q$ if and only if $G$ contains no members of $\mathbb{B}$ as a subgraph. Since the algorithm in Lemma 1 works only on TTSP graphs, we shall first reduce the decision 
problem defined on graphs to one on 2-terminal graphs. We define a set $\mathbb{B}_{T}$ of forbidden 2-terminal subgraphs as the set of all terminal-attached graphs of a graph in B. It should be noted that the set $B_{T}$ is finite since the set $\mathbb{B}$ is finite. Associated with property $Q$ on graphs, we define property $Q_{\mathrm{T}}$ on 2-terminal graphs by $\mathbb{B}_{T}: \mathbb{Q}_{T}$ is the set of all the 2-terminal graphs that contain no member of $\mathbb{B}_{T}$ as a 2-terminal subgraph.

LEMMA 2. Let $G_{T}$ be any terminal-attached graph of a graph $G$. Then $G \subseteq \mathbb{Q}$ if and only if $G_{T} \subseteq Q_{T}$.

According to Lemma 2 , it is sufficient to verify that the property $\mathbb{Q}_{\mathrm{T}}$ on 2-terminal graphs satisfies the requirements of Lemma 1. Let a set $\$$ consist of all 2-terminal graphs that belong to $\mathbb{B}_{\mathrm{T}}$ or can be obtained from a 2-terminal graph in $\mathbf{B}_{\mathrm{T}}$ by a sequence of series (of type I and type II) and parallel separations. Note that set $B_{T}$ is finite and that any separation graph has not more vertices than the original 2-terminal graph. It hence follows that set $\$$ is finite. We will show intuitively that the set of all the properties defined by a subset of $\$$ satisfies the requirements of Lemma 1. In what follows we will write $\mathbb{B}=\left\{s_{1}, s_{2}, \ldots, s_{\ell}\right\}$. Furthermore we define $S[I], \sigma_{I}(i), H_{I}(i), \sigma_{I}(J)$, and $\mu_{I}(J)$ for $i=1,2, \ldots, \ell$, and $I, J \in\{1,2, \ldots, \ell\}$ as follows:

$$
\begin{aligned}
& S[I]=\left\{s_{j}: j \leqslant I\right\} ; \\
& \sigma_{I}(i)=\left\{j: S_{j} \in S \text { and } S_{i}{ }^{*} S_{j} \in S[I]\right\} ; \\
& \mu_{I}(i)=\left\{j: s_{j} \in S \text { and } s_{i} / / S_{j} \in S[I]\right\} ; \\
& \sigma_{I}(J)=\underset{j \in J}{U} \sigma_{I}(j) ; \text { and } \mu_{I}(J)=\underset{j \in J}{=} \mu I(j) .
\end{aligned}
$$

We need some more lemmas.

LEMMA 3. Let $G_{T}$ be a 2-terminal graph such that $G_{T}=H^{*} K$ for $\mathrm{H} / \mathrm{K}$ ) for some 2-terminal graphs $\mathrm{H}$ and $\mathrm{K}$, and let $\mathrm{G}_{\mathrm{T}}$ ' be a 2-terminal graph. Then $G_{T}{ }^{\prime} \in G_{T}$ if and only if there exist 2-terminal graphs $H^{\prime}$ and $K^{\prime}$ such that $H^{\prime} \subset H^{\prime}, K^{\prime} \subset K$, and $G_{T^{\prime}}=H^{\prime *} K^{\prime}$ (or $\left.\mathrm{H}^{\prime} / / \mathrm{K}^{\prime}\right)$.

LEMMA 4. Let $\mathrm{G}_{\mathrm{T}}=\mathrm{H}^{*} \mathrm{~K}$ (or $\left.\mathrm{H} / / \mathrm{K}\right)$, and $\mathrm{S}_{\mathrm{r}} \equiv \$$. Then $\mathrm{S}_{\mathrm{r}} \subset \mathrm{G}_{\mathrm{T}}$ if and only if there exist $s_{f}, s_{g} \in \mathbb{S}$ such that $s_{x}=S_{f} s_{g}$ (or $\left.s_{f} / / s_{g}\right)$, and $s_{f} \subset^{H,} s_{g}{ }^{C K}$, 
Let $G_{T}$ be a 2 -terminal graph, and $I \subset\{1,2, \ldots, l\}$. We denote by $S[I]<G_{T}$ the proposition that $G_{T}$ contains at least one member of set $S[I]$ as a 2-terminal subgraph, and denote by $S[I] \not G_{T}$ the proposition that $G_{T}$ contains no member of $S[I]$, where the proposition $S[I] \not \subset G_{T}$ is true if $I=\phi$. Using Lemmas 3 and 4 , we can show that if $G_{T}$ is a series or parallel connection, then the solution to $G_{T}$ can be efficiently obtained from the solutions to component graphs.

LEMMA 5. Let $G_{\mathrm{s}}=\mathrm{H}^{*} \mathrm{~K}$ (of type $I$ ), $G_{\mathrm{p}}=\mathrm{H} / / \mathrm{K}$ and $I_{C}\{1,2, \ldots, \ell\}$. Then we have:

(i) $S[I] \not G_{S} \quad$ iff $\underset{J_{C I}}{\vee}\left[(S[J] \not H) \wedge\left(S\left[\sigma_{S}\left(I_{S}-J\right)\right] \not L K\right)\right]$;

and

(ii) $S[I] \not \notin \mathrm{G}_{\mathrm{p}}$ iff $\underset{\mathrm{J} \subset \mathrm{I}}{\vee}\left[(\mathrm{S}[\mathrm{J}] \not \mathrm{H}) \wedge\left(\mathrm{S}\left[\mu_{\mathrm{I}}\left(\mathrm{I}_{\mathrm{p}}-\mathrm{J}\right)\right] \not \mathrm{K}\right)\right]$, $\mathrm{J} \in \mathrm{I}_{\mathrm{p}}$

where $I_{s}=\left\{i\right.$ : there exists $s_{j \in \mathbb{B}}$ s.t. $\left.S_{i}{ }^{*} S_{j} \in S[I]\right\}$ and $I_{p}=\{i$ : there exists $s_{j} \in \mathbb{S}$ s.t. $\left.s_{i} / / S_{j} \in S[I]\right\}$.

We now have the following theorem.

THEOREM 1(2). Let $\$$ be a property on graphs defined by a finite number of forbidden (induced) subgraphs. Then the decision problem with respect to $Q$ is linear time computable for every series-parallel graph.

Proof. Let $G_{T}$ be a terminal-attached graph obtained from a series-parallel graph $G$ by designating two ends of any edge of $G$ as the terminal vertices, so that $G_{T}$ is TTSP. According to Lemma 2, the problem with respect to $Q$ on a series-parallel graph $G$ can be reduced to the problem with respect to the property $Q_{\mathrm{T}}$ on the TTSP graph $\mathrm{G}_{\mathrm{T}}$. Therefore it suffices to show that there exists a set of properties associated with $\mathbb{P}_{\mathrm{T}}$ that satisfies the requirements (i), (ii) and (iii) of Lemma 1. Consider the following set $\mathbf{p}$ of properties on 2-terminal graphs:

$\mathbb{P}=\{" S[I] ": \operatorname{I} \in\{1,2, \ldots, \ell\}\}$, where "S[I]" denotes the property defined by the set $S[I]$ of forbidden 2-terminal subgraphs: a 2-terminal graph satisfies property "S[I]" if it contains no member of $S[I]$ as a 2-terminal subgraph. We write $\mathbb{P}=\left\{\mathbb{Q}_{1}, \mathbb{Q}_{2}, \ldots, \mathbb{Q}_{k}\right\}$ where $k=2^{\ell}$. Note that set $\mathbb{P}$ is finite since $\ell$ is a constant. Then:

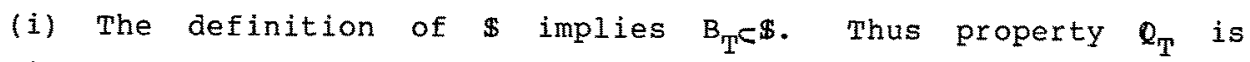
identical with the property "S[I]" with $S[I]=B_{T}$, and hence $\mathbb{Q}_{T} \in \mathbb{P}$. 
(ii) Since $\&$ is finite, one can determine in a constant time whether $S[I] \not G_{\min }$ for every $S[I]$;

(iii) It follows from Lemma 5 that for each $Q_{\mathrm{r}}$ e there exist sets of properties $\left\{\mathbb{Q}_{\mathrm{h} 1}, \mathbf{Q}_{\mathrm{h} 2}, \ldots, \mathbb{Q}_{\mathrm{ht}}\right\} \in \mathbb{R}$ and $\left\{\mathbf{Q}_{\mathrm{k} 1}, \mathbf{Q}_{\mathrm{k} 2}, \ldots\right.$, $\left.Q_{k t}\right\} \mathbb{P}$ such that

$$
\mathrm{G}_{\mathrm{T}} \in \mathbb{Q}_{\mathrm{r}} \quad \text { iff } \mathrm{V}_{i=1}^{\mathrm{V}}\left(\left(\mathrm{H} \in \mathbb{Q}_{\mathrm{hi}}\right) \wedge\left(\mathrm{K} \in \mathbb{Q}_{\mathrm{K}} \mathrm{i}\right)\right),
$$

for any $G_{T}=H * K$ (or $H / / K$ ).

\section{Edge deletion problems}

In this section we consider the minimum edge deletion problems with respect to a property $Q$ for series-parallel graphs, in which one would like to determine the minimum number of edges of a given series-parallel graph whose deletion results in a graph satisfying $Q$. We will show that the problem is also linear time computable if $Q$ can be defined by a finite number of forbidden (induced) subgraphs.

For a graph $G=(V, E)$ (or 2-terminal graph $G=(V, E, x, Y)$ ) and property $Q$ on (2-terminal) graphs, define $L(G, Q)$ as follows: if there exists a subset $E_{S}$ of $E$ such that $G-E_{S} \in \mathbb{Q}$, then $L(G, Q)$ is the minimum cardinality of such a set $E_{S}$; otherwise $L(G, Q)$ is undefined (or $\infty)$. The edge deletion problem asks $L(G, Q)$ for a given $G$ and $a$ fixed $\mathbb{Q}$. Let $\mathbb{Q}, \mathbb{Q}_{\mathrm{T}}$ and $\mathbb{R}$ be defined as in the preceding section. Then:

LEMMA 6. Let $G=(V, E)$ be a graph, and let $G_{T}=\left(V^{\prime}, E^{\prime}, x, y\right)$ be any terminal-attached graph of $G$. Then $L(G, Q)=L\left(G_{T}, Q_{T}\right)$.

Lemma 6 implies that the minimum edge deletion problem on a graph can be reduced to the same problem on a 2-terminal graph. The succeeding lemma follows from Lemmas 1 and 3.

LEMMA 7. If $G_{T}$ is a 2-terminal graph such that $G_{T}=H^{\star} K$ lof type I) (or $G_{T}=H / / K$ ) for some 2-terminal graphs $H$ and $K$, then for $\operatorname{each} \mathbb{Q}_{r} \in \mathbb{P}$

$L\left(G_{T}, Q_{r}\right)=\min _{1 \leq i \leq t}\left\{L\left(H, Q_{h i}\right)+L\left(K, Q_{k i}\right)\right\}$.

We now have the following theorem. 
THEOREM 3. Let $Q$ be a property on graphs defined by a finite number of forbidden (induced) subgraphs. Then the minimum edge deletion problem with respect to $Q$ is linear time computable for every series-parallel graph.

\section{Vertex deletion problems}

In this section we consider the vertex deletion problems with respect to a property $Q$ for series-parallel graphs, in which one would like to determine the minimum number of vertices of a given series-parallel graph whose deletion results in a graph satisfying $Q$. We will show that the vertex deletion problem is also linear time computable if $Q$ can be defined by a finite number of forbidden (induced) subgraphs.

For a graph $G=(V, E)$ and property $Q$ on graphs, define $N(G, Q)$ as follows: if there exists $V_{S} \in V$ such that $G-V_{s} \in \mathbb{Q}$, then $N(G, \mathbb{Q})$ is the minimum cardinality of such a set $V_{s}$; otherwise $N(G, Q)$ is undefined (or $\infty$ ). The vertex deletion problem asks $N(G, Q)$ for a given $G$ and a fixed $Q$.

For a 2-terminal graph $G=(V, E, x, y)$ we consider a vertex deletion problem with an additional constraint that one or both terminals should be deleted. For a property $Q$ and $m, n=1$ or 0 , define $N(G, Q, m, n)$ as follows: if there exist $V_{s} \in V$ such that (i) $G-V_{s}=Q$, (ii) $x \in V_{s}$ iff $m=1$, and (iii) $y \leqq V_{s}$ iff $n=1$, then $N(G, \mathbb{Q}, m, n)$ is the minimum cardinality of such a set $V_{s}$; otherwise $N(G, Q, m, n)$ is undefined (or $\infty)$.

Let $\mathbb{Q}, \mathbb{Q}_{T}$ and $\mathbf{R}$ be defined as in section 3 . Then we can reduce a problem on a graph to one on a 2-terminal graph as follows.

LEMMA 8. Let $G=(V, E)$ be a graph, and let $G_{T}=\left(V^{\prime}, E^{\prime}, x, y\right)$ be any terminal-attached graph of G. Then

$$
N(G, Q)=\min _{m, n \in\{0,1\}} N\left(G_{T}, \Phi_{T}, m, n\right) .
$$

\section{LEMMA 9.}

(i) If $G_{T}$ is a 2-terminal graph such that $G_{T}=H^{*} K$ (of type I) for 2-terminal graphs $H$ and $K$, then for $\mathbb{Q}_{K} \in \mathbf{P}$

$$
\begin{aligned}
& \mathrm{N}\left(\mathrm{G}_{\mathrm{T}}, \mathbb{Q}_{\mathrm{K}}, \mathrm{m}, \mathrm{n}\right)=\min \left\{\min \left\{\mathrm{N}\left(\mathrm{H}, \mathbb{Q}_{\mathrm{hi}}, \mathrm{m}, 0\right)+\mathrm{N}\left(\mathrm{K}, \mathbb{Q}_{\mathrm{K}}, 0, \mathrm{n}\right)\right\}\right. \text {, } \\
& 1 \leq i \leq t \\
& \left.\min \left\{\mathrm{N}\left(\mathrm{H}, \mathbb{Q}_{\mathrm{h} i}, \mathrm{~m}, 1\right)+\mathrm{N}\left(\mathrm{K}, Q_{\mathrm{K} i}, 1, \mathrm{n}\right)\right\}-1\right\} \text {. } \\
& \text { lis } \underline{i} \underline{t}
\end{aligned}
$$


(ii) If $G_{T}$ is a 2-terminal graph such that $G_{T}=H / / K$ for 2-terminal graphs $\mathrm{H}$ and $\mathrm{K}$, then

$N\left(G_{T}, Q_{r}, m, n\right)=\min _{1 \leq i \leq t}\left\{N\left(H, Q_{h i}, m, n\right)+N\left(K, \mathbb{Q}_{K}, m, n\right)\right\}-(m+n)$.

We now have the following theorem.

THEOREM 4. Let $Q$ be a property on graphs defined by a finite number of forbidden (induced) subgraphs. Then the minimum vertex deletion problem with respect to $Q$ is linear time computable for every series-parallel graph.

\section{Properties characterized by homeomorphic subgraphs}

In the preceding sections we showed that whenever property $Q$ is defined by a finite number of forbidden (induced) subgraphs, the decision and minimum edge (vertex) deletion problems with respect to $Q$ are all linear time computable. Although there exist graph properties whose characterizations require an infinite number of forbidden subgraphs, some of them can be characterized by a finite number of forbidden "homeomorphic subgraphs". For example, "planarity" is characterized by two forbidden homeomorphic subgraphs $\mathrm{K}_{5}$ and $\mathrm{K}_{3,3}$, while it requires an infinite number of forbidden subgraphs, i.e., all the graphs that are homeomorphic to $\mathrm{K}_{5}$ or $\mathrm{x}_{3,3}$, if one characterizes it by forbidden "subgraphs" instead of "homeomorphic subgraphs".

In this section we will show that if property $Q$ is defined by a finite number of homeomorphic subgraphs, the decision and minimum edge (vertex) deletion problems are also linear time computable.

We now present some more terminology. Let $G_{1}=\left(V_{1}, E_{1}\right)$ and $\mathrm{G}_{2}=\left(\mathrm{V}_{2}, \mathrm{E}_{2}\right)$ be any two graphs. $\mathrm{G}_{2}$ is homeomorphic to $\mathrm{G}_{1}$ if there exist mappings $\psi$ and $\theta, \psi$ from $E_{1}$ into a set of paths of $\mathrm{G}_{2}$ and $\theta$ from $v_{1}$ into $v_{2}$, such that, for each edge $(v, w) \in E_{1}$, path $\psi((v, w))$ has ends $\theta(v)$ and $\theta(w)$, and no two paths $\psi\left(\left(v_{1}, w_{1}\right)\right)$ and $x\left(\left(v_{2}, w_{2}\right)\right)$ share a vertex except possibly an end of both paths. Next let $G_{1}=\left(V_{1}, E_{1}, x_{1}, y_{1}\right)$ and $G_{2}=\left(V_{2}, E_{2}, x_{2}, y_{2}\right)$ be any two 2-terminal graphs. $G_{2}$ is 2-terminal homeomorphic to $G_{1}$ if (i) the underlying graph of $G_{2}$ is homeomorphic to the underlying graph of $G_{1}$, (ii) $x_{2}$ (or $y_{2}$ ) is virtual if and only if $x_{1}$ (or $y_{1}$ ) is virtual, and (iii) the associated mapping $\theta$ 
satisfies $\theta\left(x_{1}\right)=x_{2}$ and $\theta\left(y_{1}\right)=y_{2}$. For a 2 -terminal graph $G$ we denote by $\operatorname{Hom}(G)$ the set of all the 2-terminal graphs that are 2-terminal homeomorphic to $\mathrm{G}$.

Suppose that property $Q$ is defined by a finite set of forbidden homeomorphic subgraphs, say $B=\left\{B_{1}, B_{2}, \ldots, B_{q}\right\}$ : set $Q$ consists of all the graphs that contain no subgraph homeomorphic to any $B_{i}$, $i=1,2, \ldots, q$. As in the section 5 , let $B_{T}$ be the set of all the terminal-attached graphs of $B_{1}, B_{2}, \ldots, B_{q} \cdot$ Define the set $B_{h}$ of 2-terminal graphs as follows:

$$
\begin{aligned}
& \mathbb{B}_{\mathrm{h}}=\mathbb{B}_{\mathrm{T}} \cup \mathbf{B}_{\mathrm{I}} \cup \mathbb{B}_{I I} \cup \mathbb{B}_{I I I} \\
& \mathbf{B}_{I} \text { : the set of all 2-terminal graphs obtained from some } \\
& B_{i} \in \mathbb{B} \text { by two operations: replace any edge }(u, v) \text { of } B_{i} \\
& \text { by a new real terminal } z \text { together with two edges }(u, z) \\
& \text { and }(z, v) \text {; and designate either an arbitrary vertex of } \\
& \mathrm{B}_{i} \text { different from } z \text { or a newly added isolated virtual } \\
& \text { vertex as the other terminal; }
\end{aligned}
$$

We now define property $\mathbb{Q}_{h}$ on 2-terminal graphs by the set $\boldsymbol{B}_{h}$ of forbidden 2-terminal homeomorphic subgraphs $:$ set $\mathbb{Q}_{h}$ consists of all the 2-terminal graphs containing no 2-terminal subgraphs that are 2-terminal homeomorphic to any member of $B_{h}$. Then we have the following lemma.

LEMMA. 10. Let $G_{T}=\left(V^{\prime}, E^{\prime}, x, y\right)$ be a terminal-attached graph of a graph $G=(V, E)$. Then $G=Q$ if and only if $G_{T} \& Q_{h}$.

The following result concerning $\phi_{h}$ corresponds to Lemmas 6 and 8.

LEMMA 11. If $G_{T}$ is a terminal-attached graph of a graph $\mathrm{G}=(\mathrm{V}, \mathrm{E})$, then

(i) $L(G, Q)=L\left(G_{T}, \mathbb{Q}_{h}\right)$; and

(ii) $N(G, Q)=\min N\left(G_{T}, Q_{h}, m, n\right)$. $m, n \leqslant\{0,1\}$ 
The preceding Lemmas 10 and 11 imply that the decision problem and the minimum edge (vertex) deletion problem both with respect to property $\ell$ on a graph can be reduced to the same problems with respect to property $Q_{h}$ on a 2-terminal graph. In what follows we verify that $Q_{h}$ satisfies the requirement of Lemma 1.

We now define a new series separation in terms of its associated connection.

Definition 7. Let $\mathrm{H}=\left(\mathrm{V}_{\mathrm{H}}, \mathrm{E}_{\mathrm{H}}, \mathrm{x}, \mathrm{z}_{2}\right)$ and $\mathrm{K}=\left(\mathrm{V}_{\mathrm{K}}, \mathrm{E}_{\mathrm{K}}, \mathrm{z}_{1}, \mathrm{y}\right)$ be 2-terminal graphs. If both $z_{1}$ and $z_{2}$ are real vertices and the sum of degrees of $z_{1}$ in $K$ and $z_{2}$ in $H$ is two, then a series connection of type III of $H$ and $R$ is a 2-terminal graph $G_{S}$ obtained from the union of $\mathrm{H}$ and $\mathrm{K}$ by identifying $z_{1}$ and $z_{2}$ and replacing the two series edges incident to the identified vertex by a single edge. Then $H$ and $K$ are called series separations of type III of $G_{S}$.

From now on we write $G=H^{*} h^{K}$ if $H$ and $K$ are series separations of type I, II or III of $G$.

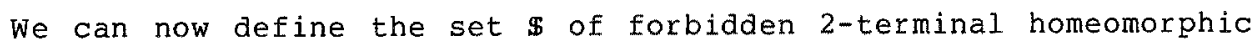
subgraphs as the set of all the 2-terminal graphs that are members of $\mathbb{B}_{h}$ or can be obtained from a member of $\mathbb{B}_{h}$ by a sequence of series and parallel separations of all the types. We write $\mathbb{S}=\left\{\mathrm{S}_{1}, \mathrm{~s}_{2}, \ldots\right.$, $\left.\mathrm{s}_{\ell}\right\}$. It should be noted that $\mathbb{B}_{\mathrm{h}}$, and hence $\mathbb{S}$, are finite sets. For $i=1,2, \ldots, \ell$ and $a$ subset $I$ of $\{1,2, \ldots, l\}$, define $S[I]$ and $\mu_{I}(i)$ as in Section 3, while define $\sigma_{I}(i)$ as follows :

$\sigma_{I}(i)=\left\{j: s_{j} \leqslant \mathbb{S}\right.$ and $\left.s_{i}{ }^{*}{ }_{h} S_{j} \in S[I]\right\}$

We write $\operatorname{Hom}\left(S_{r}\right) C_{T}$ if $G_{T}$ contains at least one member of the set Hom $\left(S_{r}\right)$ as a 2-terminal subgraph. The following lemma corresponds to Lemma 4 of section 3.

LEMMA 12. Let $G_{T}$ be a 2-terminal graph such that $G_{T}=H^{*} \mathrm{~K}$ for $\mathrm{H} / / \mathrm{K})$ where $\mathrm{H}=\left(\mathrm{V}_{\mathrm{H}}, \mathrm{E}_{\mathrm{H}}, \bar{a}, \mathrm{~b}\right)$ and $\mathrm{K}=\left(\mathrm{V}_{\mathrm{K}}, \mathrm{E}_{\mathrm{K}}, \mathrm{C}, \mathrm{d}\right)$, and let $\mathrm{S}_{\mathrm{r}}=\$$. Then Hom $\left(S_{r}\right) \subset G_{T}$ if and only if there exist $S_{f}, S_{g}=\$$ such that $s_{r}=s_{f}{ }^{k} s_{g}\left(s_{f} / / s_{g}\right)$, Hom $\left(s_{f}\right) \subset H$, and Hom $\left(s_{g}\right) \subset K$.

Using the preceding lemma, we obtain the following result, where we write Hom(S[I]) $\not G_{S}$ if $G_{S}$ contains no member of the set Hom(S[I]) as a 2-terminal subgraph.

LEMMA 13. Let $G_{s}$ and $G_{p}$ be 2-terminal graphs such that 
$G_{S}=H^{*} R$ (of type $I$ ) and $G_{p}=H / / K$, and let $I c\{1,2, \ldots, \ell\}$. Then;

(i) $\operatorname{Hom}(S[I]) \notin G_{S}$ iff

$$
\underset{J \subset I}{V}\left[(\operatorname{Hom}(S[J]) \not \nexists H) \wedge\left(\operatorname{Hom}\left(S\left[\sigma_{I}\left(I_{S}-J\right)\right]\right) \not K\right)\right] ;
$$

and

(ii) Hom (S[I]) $\not \mathrm{G}_{\mathrm{p}}$ iff

$$
\underset{J_{C I}}{v}\left[(\operatorname{Hom}(S[J]) \not \subset H) \wedge\left(\operatorname{Hom}\left(S\left[\mu_{I}\left(I_{P}-J\right)\right]\right) \not \subset K\right)\right],
$$

where

and

$I_{s}=\left\{i\right.$ : there exists $S_{j} \in \mathbb{S}$ such that $\left.s_{i}{ }^{*} S_{j} \in S[I]\right\} ;$

$I_{p}=\left\{i\right.$ : there exists $s_{j} \in S$ such that $\left.s_{i} / / S_{j} \leqslant S[I]\right\}$.

We now have the following theorem by Lemmas 1 and 10-13.

THEOREM 5. Let $\mathbb{Q}$ be a property on graphs defined by a finite number of forbidden homeomorphic subgraphs. Then the decision problem and the minimum edge (vertex) deletion problem, both with respect to Q, are linear time computable for every series-parallel graph.

Acknowledgement. This work was partly supported by the Grant in Aid for Scientific Research of the Ministry of Education, Science and Culture of Japan under Grant: Cooperative Research (A) 435013 (1980).

REFERENCES

[1] A. V. Aho, J. E. Hopcroft and J. D. Ullman, The Design and Analysis of Computer Algorithms, Addison-Wesley, Reading, Mass. 1974 .

[2] M. Boulala and J. Uhry, Polytope des independants d'un graphe series-parallel, Discrete Math., 27, pp. 225-243 (1979).

[3] G. Chartrand, D. Geller and S. Hedetniemi, Graphs with forbidden subgraphs, J. of Combinatorial Theory, 10, pp. 12-41 (1971).

[4] R. J. Duffin, Topology of series parallel networks, J. Math. and Appli. 10, pp. 303-318 (1965).

[5] J. Edmonds, Paths, trees, and flowers, Canad. Math. 17, pp. 449-467 (1965).

[6] S. Even and 0 . Kariv, An $O\left(n^{2.5}\right)$ algorithm for maximum matching in general graphs, Proc. IEEE 16 th symp. on Focs, pp. 100-112 (1975).

[7] M. R. Garey, D. S. Johnson and L. Stockmeyer, Some simplified NP-complete graph problems, Theoretical Computer Science, 1, pp. 237-267 (1976).

[8] J. E. Hopcroft and R. E. Tarjan, Dividing a graph into triconnected components, SIAM J. Comput., 2, 3, pp. 135-158 (1973). 
[9] F. O. Hadlock, Finding a maximum cut of a planar graph in polynomial time, SIAM J. Comput., 4, pp. 221-225 (1975).

[10] F. Harary, Graph Theory, Addison-Wesley, Reading, Mass. 1969.

[11] S. T. Hedetniemi, Hereditary properties of graphs, J. Combinatorial Theory (B), 14, pp. 94-99 (1973).

[12] M. S. Krishnamoorthy and N. Deo, Node-deletion NP-complete problems, SIAM J. Comput., 8, 4, pp. 619-625 (1979).

[13] D. G. Kirkpatrick and P. Hell, On the completeness of a generalized matching problem, Proc. 10th ACM Symp. on Theory of Computing (1978).

[14] T. Kikuno, N. Yoshida and $Y$. Kakuda, Dominating set in planar graphs, Tech. Report AL79-9, Inst. Elect. Commun. Eng. Japan, pp. $21-30$ (1979).

[15] J. M. Lewis, on the complexity of the maximum subgraph problem, Proc. of the 10th ACM Symp. on Theory of Computing, pp. 265-274 (1978).

[16] C. L. Monma and J. B. Sidney, Sequencing with series-parallel procedure constraints, TR 347, Sch. of OR/IE, Cornell university (1979).

[17] E. F. Moore and C. E. Shannon, Reliable circuits using reliable relays I-II, J. Franklin Inst., 262, 3, p. 191, and 4, p. 281 (1956).

[18] T. Nishizeki and N. Saito, Necessary and sufficient condition for a graph to be three-terminal series-parallel-cascade, J. of Combinatorial Theory B, 24, 3, pp. 344-361 (1978).

[19] T. Nishizeki, K. Takamizawa and N. Saito, Algorithms for detecting series-parallel graphs and D-charts, Trans. of Inst. Elect. Commun. Eng. Japan, 59, 3, pp. 259-250 (1976).

[20] N. Tomizawa, on a specialization sequence from general matroids to ladder graphs with special emphasis on the characterization of ladder matroids, RAAG Research Notes, Third Series, No. 191 (1973).

[21] J. Valdes, R. E. Tarjan and E. L. Lawler, The recognition of series-parallel digraph, Eroc, of lith Ann. ACM Symp. on Theory of Computing, 1979.

[22] T. Watanabe, T. Ae and A. Nakamura, On the node cover problem of planar graphs, Proc. of 1979 ISCAS, pp. 78-81 (1979).

[23] M. Yannakakis, Node- and edge-deletion. NP-complete problems, Proc of the 10th ACM Symp. on Theory of Computing, pp. 253-264 (1978). 